\title{
EDUCAUTISM: Um sistema personalizável para o apoio à educação de criaças diagnosticadas com o transtorno do espectro autista
}

\author{
Ivan Andreis, Sandro José Rigo Universidade \\ do Vale do Rio dos Sinos \\ ivann.andreis@gmail.com, rigo@unisinos.br
}

\begin{abstract}
Resumo. Desde o século passado são estudadas diferentes formas de auxiliar e apoiar o desenvolvimento de crianças diagnosticadas com o transtorno do espectro autista. Estas crianças muitas vezes demonstram pouco interesse em assuntos variados e denotam uma fixação maior em tópicos específicos. Eeste trabalho apresenta um sistema que auxilia na resolução do problema de uso e adaptação à softwares de apoio à educação por parte das crianças diagnosticadas com o transtorno. Este sistema oferece uma nova proposta de interação ao usuário, com uma interface totalmente personalizável, de acordo com o perfil da criança, que leve em consideração sua sensibilidade a cores, imagens, sons e outros aspectos relevantes. Pais e responsáveis poderão customizar esta interface, buscando manter o foco, interesse e motivação da criança em realizar as atividades, evitando que a mesma repudie alguma característica que lhe traga irritabilidade ou cause uma crise nervosa, característica comum entre os indivíduos portadores do espectro. Com isso, o trabalho em questão visa a contribuir com a melhora na qualidade de vida dos usuários e respectivos familiares. Foi desenvolvido um protótipo com o modelo, cuja avaliação permitiu identificar diversos aspectos positivos neste sentido de apoio à qualidade de interação.
\end{abstract}

Palavras-chave: Transtorno do Espectro Autista, Autismo, Personalização, Customização.

Abstract. Since the last century different ways of assisting and supporting the development of children diagnosed with autism spectrum disorder have been studied. These children often show little interest in different subjects, and a greater fixation on specific topics. The solution proposed in this work focuses on the development of a system that helps to solve the problem of usability in an educational software, from children diagnosed with the disorder. This systems aims, therefore, to offer a new proposal of interaction to the user, taking into account their sensitivity to colors, images, sounds, etc., and providing a fully customizable interface according to the child's profile. Parents and tutors will be able to customize this interface, aiming to maintain the child's focus, interest and motivation in performing the activities, and avoiding that the child repudiates some characteristic that causes irritability or causes a nervous crisis, a common characteristic among individuals with the spectrum. Finally, this work aims to contribute to the scientific community, especially in the autism area, with the improvement in the quality of life of the users and their relatives..

Keywords: Autistic Spectrum Disorder, Autism, Personalization, Customization.

\section{INTRODUÇÃ̃O}

Nos últimos anos, vem sendo discutidas formas de aliar o uso da tecnologia para melhorar o desenvolvimento educacional de crianças portadoras do transtorno do espectro autista. $\mathrm{O}$ autismo afeta o desenvolvimento de habilidades essenciais para os indivíduos, como suas capacidades de comunicação e interação social, além destes demonstrarem um menor interesse por determinados assuntos e em contrapartida uma fixação exagerada em outros tópicos e objetos, o que vem a diminuir e dificultar o seu processo de aprendizado (Pauline A. Filipek et al., 1999). Embora o autismo tenha sido descrito pela primeira vez há 50 anos atrás, por Kanner (1943), o entendimento mais completo dessa desordem só 
passou acontecer de forma efetiva nas duas últimas décadas, mas ainda é um tema sendo estudado e em constante evolução (Fitzgerald, 2011).

Enquanto nos modelos tradicionais de educação o ensino é baseado principalmente na comunicação verbal, indivíduos com autismo geralmente encontram sérias dificuldades para desenvolver seu aprendizado com este tipo de abordagem. Estes indivíduos tendem a reagir negativamente, com ataques de stress e irritação que podem atrapalhar e interromper até mesmo totalmente o processo de aprendizado e desenvolvimento educacional (Jordan RR, Powell S D.,1995). Uma alternativa para endereçar este problema e amenizar os efeitos negativos do espectro no processo de aprendizado dos indivíduos autistas são as Tecnologias de Informação e Comunicação. As estratégias de aprendizado baseadas no uso destas tecnologias trouxeram um progresso significativo para a área. Entretanto, atualmente nota-se uma maior disponibilidade somente de conteúdo de ensino padronizado, o que acaba não sendo a melhor abordagem de ensino às crianças autistas, considerando que cada indivíduo tem suas próprias necessidades e interesses (Margarida Lucas da Silva et al.2014).

Analisando os trabalhos relacionados a este tema, nota-se a falta de uma proposta na qual as crianças diagnosticadas com o transtorno do espectro autista possam usufruir de uma interface totalmente personalizável e de fácil uso, para assim evitarem crises nervosas e se sentirem motivadas a continuarem fazendo o uso da mesma e melhorando seu nível educacional. Os principais trabalhos pesquisados estão focados mais no conteúdo em si das atividades do que na oferta de uma interação humano-computador simplificada, em sua maioria.

O objetivo deste trabalho é criar um modelo de interação humano-computador, constituído de uma interface personalizável, para uso em dispositivos móveis e suporte à tecnologia Touchscreen, evitando crises nervosas por parte do usuário. Com isso, o trabalho em questão visa a contribuir com a melhora na qualidade de vida dos usuários e respectivos familiares. Foi desenvolvido um protótipo com o modelo, cuja avaliação permitiu identificar diversos aspectos positivos neste sentido de apoio à qualidade de interação.

Este artigo está organizado como segue. São apresentados elementos sobre embasamento teórico envolvendo autismo e tecnologias aplicadas à esta questão na seção 2. Os trabalhos relacionados estudados e sua análise são apresentados na seção 3. A seção 4 descreve o sistema desenvolvido, enquanto que a seção 5 apresenta as avaliações realizadas. As conclusões são descritas na seção 6.

\section{Tecnologia e autismo}

O Transtorno do Espectro Autista é definido como uma perda significativa nas interações sociais por parte do indivíduo, caracterizada por um dos seguintes itens: problemas com o uso de comportamento nãoverbal; pouco contato visual com outros indivíduos; dificuldade de desenvolver relações com colegas; falta de vontade de compartilhar interesses e prazeres com terceiros; falta de reciprocidade emocional e social. Para confirmar o diagnóstico, o indivíduo também deve apresnetar uma deficiência na categoria de comunicação como: atraso no desenvolvimento da fala; problemas com iniciar ou manter a fala; uso repetitivo ou estereotipado de linguagem; dificuldade de ter espontaneidade ao brincar de "imaginar" um cenário com colegas. Finalmente, é necessário ter um comportamento de padrão repetitivo ou estereotipado de: preocupação excessiva com um interesse restrito; preservação da mesmice; função motora repetitiva ou estereotipada; preocupação persistente com partes específicas de objetos. Os sintomas também devem ser notados antes dos três anos de idade. Diversos estudos têm demonstrado que softwares e tecnologia tendem a ser bem recebidos como ferramentas de aprendizado por indivíduos portadores do transtorno do espectro autista (Putnam, 2008). Além disso, pesquisas também comprovam a efetividade de treinamentos baseados em computadores para ensinar uma variedade de habilidades às crianças com esse transtorno (Bernard-Opitz et al., 2004). 
Alguns dos motivos para esta aceitação e efetividade demonstrados pelas pesquisas incluem: a) Programas de computador acomodam a necessidade de uniformidade por serem previsíveis e familiares (Williams et al.,2002). b) Tarefas podem ser repetidas facilmente sem grandes mudanças. Os programas não ficam impacientes por terem de repeti-las (Williams et al.,2002) e podem ser implementados para reforçar as atividades constantemente (Whalen et al.,2006). c) A maioria dos programas e treinamentos baseados no uso de computadores eliminam as interações sociais complexas com terceiros e permitem ao usuário autista trabalhar na sua própria velocidade (Powell, 1996). d) Softwares educacionais para computadores pessoais podem fornecer um ambiente de aprendizado estruturado e individual, geralmente necessário para uma criança autista aprender um novo tópico com efetividade (Williams et al.,2002).

Desta forma, pode-se destacar que softwares desenvolvidos para dispositivos tecnológicos podem prover ferramentas de aprendizado práticas e acessíveis que ampliem os métodos de ensino off-line, permitindo acesso das crianças e dos adultos de casa ou da escola, eliminando a necessidade de uma abordagem individualizada entre a criança e o seu instrutor (Putnam, 2008).

O Departamento de Saúde do Reino Unido criou uma metodologia com certas recomendações para o desenvolvimento de interfaces para pessoas com dificuldade de aprendizado (Department of Health (UK), 2009). Abaixo são destacadas as regras definidas:

Regra 1: Cada ideia necessita de ambos, palavras e imagens, sendo que ambos são importantes;

Regra 2: Imagens e palavras devem ser posicionadas lado a lado, pois isto auxilia mais pessoas a compreenderem a informação;

Regra 3: Defina claramente qual imagem suporta qual parte do texto;

Regra 4: Imagens devem ser fáceis de entender;

Regra 5: Imagens devem ser posicionadas à esquerda;

Regra 6: Imagens podem ser desenhos, fotografias ou outros tipos de imagens;

Regra 7: Garanta que as imagens têm o tamanho máximo possível;

Regra 8: Palavras devem ser fáceis de compreender;

Regra 9: Se utilizar palavras difíceis, explique o que elas significam com palavras fáceis;

Regra 10: Palavras devem ser posicionadas à direita;

Regra 11: Palavras devem ser escritas claramente, com uma fonte como o Arial;

Regra 12: Palavras devem ser escritas com tamanho grande. Tamanho de fonte de pelo menos 14 é ideal;

Regra 13: Cada frase deve ser o menor possível. Mais do que 15 palavras por frase torna difícil a leitura;

Regra 14: Cada documento deve ser curto. Mais do que 20 páginas passa a ser muito extenso.

Outras recomendações específicas para interfaces de usuário projetadas para pessoas com o Transtorno do Espectro Autista incluem (Pavlov, 2014): a) Apresentação: Uso de contraste entre a fonte e o plano de fundo. Uso de cores leves. Garantir que o texto está claramente separado do restante. Uso de gráficos simples. Uso de fontes sans-serif claras. Evitar o uso de cores brilhantes, imagens ao fundo, elementos de pop-up e distrações, elementos se destacando em relação aos outros, scrolling horizontal, e transposição de imagens transparentes e texto. b) Navegação e carregamento de páginas: Procure utilizar navegação simples e clara. Indique claramente em cada página onde o usuário está. Suporte navegação por mouse e teclado. Permita o uso de botões do navegador. Páginas devem carregar rapidamente. Use indicadores visuais para atividades que levam mais tempo. Ofereça um botão de Ajuda. Evite o uso de menus complexos. c) Interação: Busque o design por simplicidade e poucos elementos na tela. Procure ter somente uma barra de ferramentas. Use botões grandes e claros, com ambos textos e ícones. Dê instruções curtas de uso a cada etapa (Mintz, 2013). Evite interfaces confusas (Mintz, 2013), uso de muitos ícones V. $16 \mathrm{~N}^{\mathrm{o}} 1$, julho, 2018 
coloridos, e evite botões sem texto, exceto para ações populares como por exemplo "Voltar". d) Personalização: Permita ao usuário a personalização do tipo de fonte e tamanho, espaçamento de linhas e temas para o texto de fundo e cores de primeiro plano.

\section{TRABALHOS RELACIONADOS}

Foram estudados trabalhos relacionados ao uso de interfaces para a apoio à educação de crianças com necessidades especiais, que fossem personalizáveis de acordo com as necessidades do usuário final. Não foram encontrados trabalhos que aliassem todas as características de personalização e usabilidade propostas, portanto a avaliação dos trabalhos limitou-se aos seguintes critérios: a) Conteúdo Multimídia: Se a solução oferece a possibilidade de manter conteúdos multimídia para a criança, como animações, jogos, etc. b) Conteúdo Personalizável: Se a solução oferece a possibilidade de personalizar seu conteúdo, ou se somente conteúdo padrão / pré-definido é entregue. c) Layout Personalizável: Se a solução permite a personalização do Layout da interface, como imagem e cor de plano de fundo, cores dos menus, ícones, avatares, etc. d) Configuração da Ferramenta: Se a solução permite a configuração da ferramenta, pelo usuário final ou pelo seu tutor / professor / responsável. e) Design centrado no usuário: Se a solução permite que o design da mesma seja focado à nível de perfil de usuário, definindo diferentes designs de acordo com a preferência do mesmo. f)Touchscreen/mobile: Se a solução está disponível para uso em plataforma mobile e, portanto, Touchscreen, ou se somente web/desktop.

A Tabela 1 apresenta esses critérios aplicados aos trabalhos selecionados, possibilitando uma melhor análise comparativa. Para uma melhor apresentação dos trabalhos na tabela, os mesmos estão representados conforme segue: Trabalho 1: Gianluca De Leo and Gondy Leroy. 2008. Smartphones to facilitate communication and improve social skills of children with severe autism spectrum disorder: special education teachers as proxies. Trabalho 2: A. Ismail, N. Omar and A. Mohd Zin, "Developing learning software for children with learning disabilities through Block-Based development approach,". Trabalho 3: Robert R. Morris, Connor R. Kirschbaum, and Rosalind W. Picard. 2010. Broadening accessibility through special interests: a new approach for software customization. Trabalho 4: M. R. Rahman et al., "A-class: A classroom software with the support for diversity in aptitudes of autistic children,". Trabalho 5: Margarida Lucas da Silva, Daniel Gonçalves, Tiago Guerreiro, Hugo Silva, A Web-based Application to Address Individual Interests of Children with Autism Spectrum Disorders.

Tabela 1. Comparação dos critérios entre os trabalhos

\begin{tabular}{|l|c|c|c|c|c|c|}
\hline & Tr. 1 & Tr. 2 & Tr. 3 & Tr. 4 & Tr. 5 & "Educautism" \\
\hline $\begin{array}{l}\text { Conteúdo } \\
\text { Multimídia }\end{array}$ & Sim & Sim & Sim & Sim & Sim & Sim \\
\hline $\begin{array}{l}\text { Conteúdo } \\
\text { personalizável }\end{array}$ & Sim & Não & Sim & Sim & Sim & Sim \\
\hline $\begin{array}{l}\text { Layout } \\
\text { Personalizável }\end{array}$ & Não & Não & Não & Não & Sim & Sim \\
\hline $\begin{array}{l}\text { Configuração da } \\
\text { ferramenta }\end{array}$ & Não & Sim & Sim & Não & Sim & Sim \\
\hline $\begin{array}{l}\text { Design centrado } \\
\text { no usuário }\end{array}$ & Não & Não & Não & Não & Sim & Sim \\
\hline $\begin{array}{l}\text { Touchscreen I } \\
\text { mobile }\end{array}$ & Sim & Não & Não & Não & Não & Sim \\
\hline
\end{tabular}

Analisando a tabela comparativa dos trabalhos relacionados a proposta, nota-se algumas semelhanças entre as soluções. Todos softwares existentes voltados ao apoio ao ensino de crianças portadoras do transtorno fornecem conteúdo multimídia, ao mesmo tempo que a maioria também possui seu conteúdo personalizável. Entretanto, poucos trabalhos oferecem soluções nas quais o próprio layout é personalizável e o design da ferramenta seja centrado no usuário. Além disso, nenhum dos trabalhos apresentados faz uso de tecnologia mobile / Touchscreen ao mesmo tempo que ofereça as demais características de configuração e personalização. É neste ponto que o Educautism procura englobar e fornecer à criança 
portadora de autismo e aos pais e responsáveis uma proposta de solução que tenha abrangência à todas as características citadas na Tabela 1 .

\section{SISTEMA PROPOSTO}

O Educautism constitui-se de uma interface que visa apoiar o processo de desenvolvimento e educação de crianças autistas. A proposta principal da solução passa por prover um software com grau elevado de personalização em sua interface, de maneira que os pais / responsáveis / tutores da criança possam adequálo o máximo possível às preferências e interesses do indivíduo, a fim de motivar o seu uso e auxiliar na efetividade no processo de aprendizado durante a realização das tarefas, que podem ser constituídas por jogos educativos, animações, etc.

O modelo de prioridades pensado para a solução passa pelo foco em uma interface que evite ao máximo a crise nervosa de uma criança autista enquanto ela realiza as tarefas designadas. Isto porque o desencadeamento de uma crise de irritação é o fator mais desmotivante para que o indivíduo continue realizando suas tarefas. Em segundo lugar, procura-se prover uma interação descomplicada, assim como em todas as interfaces, buscando-se a simplicidade para diminuir a complexidade do seu uso e aumentar a praticidade na realização das atividades. Por último, mas não menos importante, faz -se necessário motivar o uso do software por parte da criança portadora do transtorno do espectro autista. Isso porque mesmo trabalhando para evitar crises nervosas e prover uma interação descomplicada, indivíduos com autismo possuem preferências e interesses específicos, elevando a necessidade de alto nível de personalização da interface por parte dos responsáveis, aumentando a motivação quanto ao seu uso.

Já o módulo de perfis engloba os aspectos relacionados a personalização do conteúdo, do layout e configuração da solução. Aspectos como a cor de fundo da interface, o papel de parede (se aplicável), o conteúdo das tarefas disponíveis, quais emoticons, avatares, ícones e sons estarão disponíveis para cada usuário (criança) específico. Desta forma, os pais ou responsáveis, conhecedores das preferências e interesses de cada indivíduo autista podem personalizar a interface o máximo possível para motivar o seu uso, aumentar o interesse por parte da criança e evitar possíveis crises nervosas. A proposta do módulo de configuração passa por proporcionar, de maneira simplificada e prática, a personalização da interface por parte dos responsáveis. Desta forma, os tutores, pais, etc. podem definir a aparência da tela principal do sistema, do menu e das atividades específicas de maneira a configurar a interface com as características, interesses e preferências do usuário final, a criança com autismo. As Figuras 1 e 2 demonstram o módulo de perfis de usuários e o módulo de configuração.

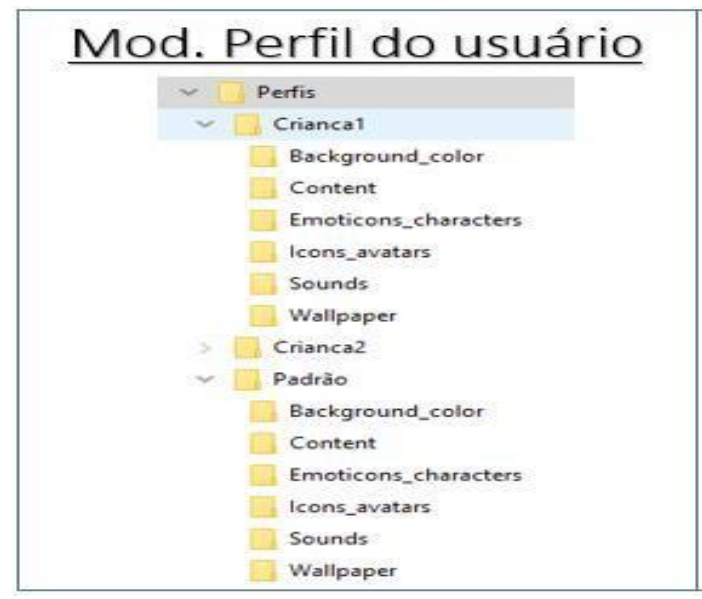

Figura 1. Módulo de perfis de usuários

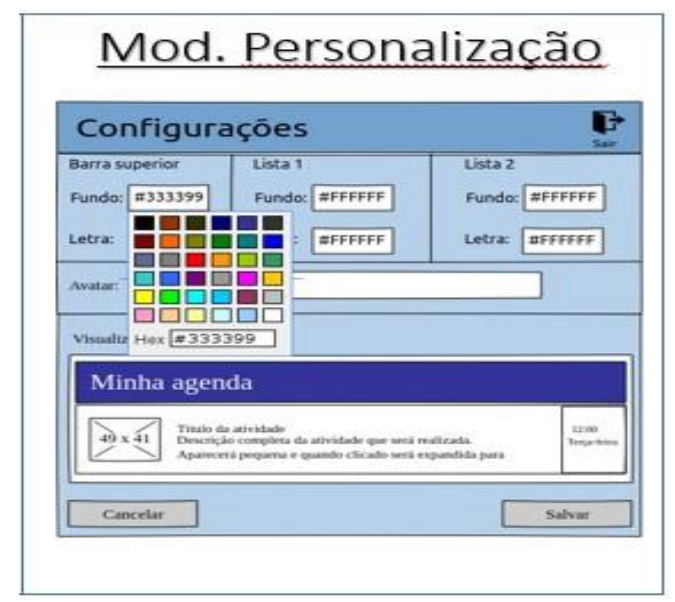

Figura 2. Layout - módulo de configuração da ferramenta

O módulo de recomendação de conteúdo irá determinar quais atividades estarão presentes para cada usuário diferente do sistema. Isto ocorre de acordo com o perfil do usuário, já que o mesmo é configurado

V. $16 \mathrm{~N}^{\mathrm{o}} 1$, julho, 2018 
pelo pai ou responsável, que determina as preferências de cores de fundo, uso ou não de sons, avatares e ícones de interesse da criança, etc. Desta forma, o módulo de recomendação de conteúdo irá buscar dentre o pacote completo de atividades disponíveis quais as atividades que se encaixam com as preferências do indivíduo autista, de modo a somente ofertar as atividades que se enquadram com o perfil do usuário. Vale ressaltar que os módulos de perfil e de configuração também são utilizados para a personalização da interface como um todo, porém é no módulo de recomendação de conteúdo que é feita a escolha das atividades que a criança autista terá designadas para realização.

A fim de validar a solução proposta, foi desenvolvido um protótipo contendo suas funcionalidades. A aplicação web desenvolvida permite ao usuário personalizar a interface quanto ao seu layout, permitindo a mudança de cor de fundo em tempo real, além de ser multi-idiomas (português e inglês), possibilitar a definição de um avatar de preferência do usuário e a seleção das categorias de jogos educativos de interesse da criança autista. O objetivo do protótipo, portanto, é proporcionar uma experiência de usabilidade que motive o uso por parte do usuário, provendo uma interação descomplicada e desta forma mantendo a criança engajada nas atividades disponibilizadas. Foi desenvolvida uma aplicação web responsiva, utilizando-se o Framew ork Web PHP CodeIgniter, e banco de dados MySQL, tendo seu layout adaptado para ser utilizado em computadores, tablets e celulares sem comprometer a usabilidade. A aplicação foi testada usando um smartphone com sistema operacional Android versão 6.0.

Para facilitar a personalização do layout, o pai, responsável ou a própria criança podem facilmente alterar a cor de fundo de sua preferência, mesmo durante a execução dos jogos educativos selecionados. Além disso, por padrão, antes e após um usuário novo efetuar o cadastro, a cor de fundo da tela é pré-definida como azul claro, de forma a evitar crises nervosas por parte da criança autista, já que na maioria dos casos o azul é uma cor neutra que leva o indivíduo diagnosticado com o autismo ao equilíbrio e a momentos de calma, ajudando nos episódios de sobrecarga sensorial (Manual da Pedagogia das Cores, 2012). A figura 03, item a, mostra o componente disponível para esta configuração. A opção de personalizar as categorias de jogos educativos disponíveis deve ser acessada pelos pais ou responsáveis previamente, e fica memorizada, assim como a cor de fundo, após cada evento de logout e logon na aplicação. A figura 03, item b, mostra a tela de seleção de categorias para o usuário.

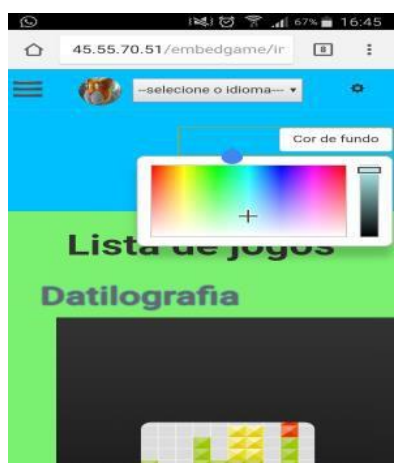

a) Layout

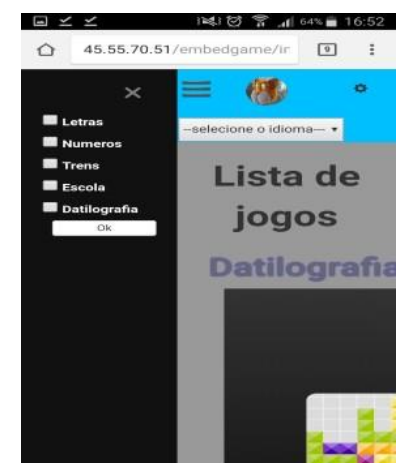

b) Conteúdo

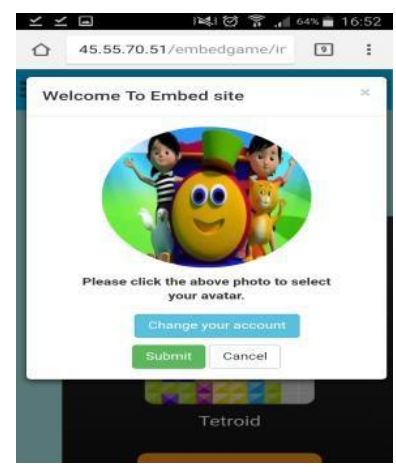

c) avatar

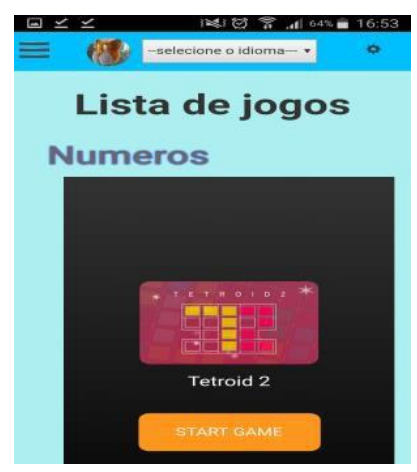

d) Tela de atividades

Figura 03. Personalização dos elementos e tela de atividades

Outra opção disponível, a personalização do avatar, é habilitada assim que o usuário clica sobre a imagem do avatar corrente. Na mesma tela, pode-se alterar a senha atual para login, caso o usuário deseje, e por fim salvar as alterações, como pode ser visto na figura 03, item c. A figura 03, item d traz um esboço de atividades para a criança realizar de acordo com a categoria escolhida anteriormente no menu lateral. 


\section{AVALIAÇÃO}

Três métodos de avaliação foram utilizados. O primeiro se deu através de um levantamento antecipado para buscar recomendações prévias ao desenvolvimento do protótipo. Além disso, foi utilizada a estratégia de validação por cenários, que tem sido utilizada pela comunidade científica para avaliação de sistemas sensíveis ao contexto (DEY; ABOWD; SALBER, 2001) e sistemas ubíquos (SATYANARAYANAN, 2001). Sendo assim, foi criado um cenário, detalhado na subseção a seguir, para simular uma situação possível imaginando o contexto da realização de atividades educativas por parte de uma criança diagnosticada com o transtorno do espectro autista. Por final, uma pesquisa qualitativa foi enviada para cinco indivíduos com experiência relevante na área de autismo a fim de validar a aceitação do protótipo proposto.

\subsection{Levantamento antecipado}

Uma das maneiras existentes de aumentar a eficiência do desempenho de sistemas passa por um levantamento prévio, antes do início do processo de desenvolvimento. Testes antecipados podem detectar erros ou falhas antes que se tornem mais caros para solucionar (UNGER; CHANDLER, 2009, p. 226). Antes do início do desenvolvimento do protótipo foi feito um levantamento de alguns requisitos e características a serem consideradas na interface do Educautism. Este processo foi realizado com dois indivíduos autistas, que além de terem sido diagnosticados com o espectro, também possuem filhos posteriormente diagnosticados com o transtorno. Com isso, foi possível tirar proveito das recomendações de duas pessoas experientes na área do autismo. Estas, além da vivência com o transtorno em si, também são atuantes na área de desenvolvimento de sistemas. Abaixo, são listados os principais pontos e recomendações levantados na entrevista: a) Posicionar figuras à esquerda e o texto à direita nas atividades propostas; b) Uso de frases de instrução curtas, não sublinhadas; c) Evitar muita informação/texto na mesma tela; d) Oferecer alternativas para evitar crises nervosas caso o usuário esteja estagnado; e) Oferecer tarefas de acordo com humor diário do usuário; f) Evitar o uso de sons e avisos sonoros; g) Utilizar emoticons no lugar de rostos reais para expressão de emoções; h) Possibilitar escolha de instruções das atividades via voz ou imagens + texto, nunca ambos; i) Utilizar por padrão ícones / avatares de interesse comum entre indivíduos autistas (por exemplo, Thomas o trem, dinossauros, etc.); j) Evitar contraste alto entre o plano de fundo e o texto da tela;

Esta etapa, portanto, ajudou consideravelmente no planejamento prévio do desenvolvimento do protótipo funcional.

\subsection{Cenário de uso}

O objetivo deste cenário é simular uma situação na qual uma criança autista iria utilizar o Educautism, buscando-se validar a sua eficiência. Abaixo encontra-se sua descrição, que foi simulada adequadamente:

"João está procurando uma alternativa para prover conhecimento ao seu filho, Marcos, diagnosticado com o transtorno do espectro autista, de alto funcionamento. Ao utilizar o Educautism, João começa a adaptar/personalizar a interface da aplicação de acordo com as preferências e interesses de Marcos, alterando a cor de fundo do layout para laranja, pois ele sabe que seu filho fica bemhumorado quando em contato com esta cor. Além disso, João altera a linguagem da interface para inglês, pois ele sabe que Marcos não se incomoda quando em contato com palavras novas e quer que o mesmo vá praticando e aprendendo uma segunda língua, além do português. Em seguida, João edita o avatar do usuário criado para Marcos para incluir a figura de Bob, o trem, desenho favorito de seu filho e de muitas outras crianças autistas. Por fim, João escolhe as categorias de jogos que Marcos terá à disposição para realizar as atividades educativas, optando por jogos de datilografia e matemática, preferidos do seu filho. Agora, Marcos está acessando a interface diretamente com as configurações previamente realizadas pelo seu pai. Ele realiza as atividades com bom humor e não demonstra irritabilidade com nenhum aspecto da interface, mantendo seu engajamento e procurando realizá-las até o final, deste modo adquirindo um 
maior conhecimento matemático e uma maior habilidade de digitação. '”

\subsection{Avaliação de Aceitabilidade}

Para a avaliação de aceitabilidade foi utilizado o método de avaliação Technology Acceptance Model (TAM), proposto por Davis (1989) e ampliado por Yoon e KIM (2007), usando uma escala de Likert (1932). A avaliação foi realizada com cinco voluntários, sendo estes três indivíduos diagnosticados com o transtorno do espectro autista e dois indivíduos que trabalham ativamente em programas de inclusão de pessoas autistas em uma empresa privada do ramo de tecnologia da informação. Além diss o, dentre os três voluntários que possuem o transtorno, todos estudam ou estudaram na área de ciência da computação e trabalham na área de desenvolvimento de sistemas, sendo que dois destes possuem filhos também diagnosticados com autismo. Dentre as duas voluntárias que trabalham com inclusão de autistas no mercado de trabalho, uma está em formação na área de psicologia e a outra já concluiu sua graduação na área. Outro ponto a se destacar é a experiência que os indivíduos autistas com filhos autistas puder am trazer para o processo de avaliação. Ambos citaram que já utilizavam diferentes aplicativos educacionais para designar atividades de aprendizado para seus filhos. Dessa forma puderam contribuir com uma série de feedbacks quanto aos aspectos positivos e aspectos a serem melhorados no protótipo desenvolvido. A tabela 02 abaixo traz os detalhes quanto as perguntas de validação enviadas posteriormente aos voluntários via questionário, assim como suas respectivas respostas no modelo TAM.

Tabela 02. Resultado da Avaliação

\begin{tabular}{l|c|c|c|c|c}
\hline \multicolumn{1}{c|}{ Declaração / Resultados } & $\begin{array}{c}\text { Discordo } \\
\text { totalmente }\end{array}$ & $\begin{array}{c}\text { Discordo } \\
\text { parcialmente }\end{array}$ & $\begin{array}{c}\text { Não concordo } \\
\text { nem discordo }\end{array}$ & $\begin{array}{c}\text { Concordo } \\
\text { parcialmente }\end{array}$ & $\begin{array}{c}\text { Concotardo } \\
\text { totalmente }\end{array}$ \\
\hline $\begin{array}{c}\text { 1. Você se considera um usuário experiente } \\
\text { de interfaces web? }\end{array}$ & $0 \%(0)$ & $0 \%(0)$ & $0 \%(0)$ & $20 \%(1)$ & $80 \%(4)$ \\
\hline 2. A interface mobile é fácil de ser utilizada? & $0 \%(0)$ & $0 \%(0)$ & $0 \%(0)$ & $20 \%(1)$ & $80 \%(4)$ \\
\hline $\begin{array}{c}\text { 3. As atividades disponibillizadas são } \\
\text { educativas? }\end{array}$ & $0 \%(0)$ & $0 \%(0)$ & $20 \%(1)$ & $20 \%(1)$ & $60 \%(3)$ \\
\hline $\begin{array}{c}\text { 4. Os tipos de atividade e o layout da } \\
\text { interface são personalizáveis? }\end{array}$ & $0 \%(0)$ & $0 \%(0)$ & $0 \%(0)$ & $60 \%(3)$ & $40 \%(2)$ \\
\hline $\begin{array}{c}\text { 5. A interface proposta é responsiva, } \\
\text { personalizável e centrada no usuário? }\end{array}$ & $0 \%(0)$ & $0 \%(0)$ & $0 \%(0)$ & $20 \%(1)$ & $80 \%(4)$ \\
\hline $\begin{array}{c}\text { 6. Você considera o objetivo da interface } \\
\text { relevante para a sociedade? }\end{array}$ & $0 \%(0)$ & $0 \%(0)$ & $0 \%(0)$ & $0 \%(0)$ & $100 \%(5)$ \\
\hline \begin{tabular}{l} 
7. O protótipo atende a sua proposta? \\
\hline Média
\end{tabular} & $0 \%(0)$ & $0 \%(0)$ & $0 \%(0)$ & $0 \%(0)$ & $100 \%(5)$ \\
\hline
\end{tabular}

A validação foi realizada com um número reduzido de pessoas e ações, o que no caso de uma pesquisa de natureza quantitativa poderia tornar o resultado tendencioso. Porém, como se trata de uma validação através de uma pesquisa de natureza qualitativa, o número de cinco participantes é considerado adequado, considerando-se que todos os envolvidos têm ampla experiência com o tema, incluindo-se três indivíduos diagnosticados com o mesmo, além de dois destes também possuírem filhos autistas que utilizam softwares educacionais com o mesmo propósito.

Como resultado da pesquisa de validação, podemos destacar que a grande maioria dos voluntários se considera um usuário experiente de interfaces web, além de considerar a interface mobile fácil de ser utilizada. Além disso, uma pessoa se mostrou indiferente quanto as atividades disponibilizadas serem educativas, enquanto todos concordaram plenamente que o objetivo da interface é de extrema relevância para a sociedade. Quanto a sua personalização, vale destacar que mais da metade dos questionados concorda parcialmente que os tipos de atividade e o layout são personalizáveis. Quatro indivíduos concordam totalmente que a interface é responsiva e centrada no usuário. Por fim, ressalta-se que todos voluntários que participaram do processo de avaliação concordam plenamente que o protótipo atende sua proposta inicial.

\section{CONCLUSÃO}

Este trabalho detalhou o desenvolvimento de um sistema com foco na educação de crianças diagnosticadas

V. $16 \mathrm{~N}^{\mathrm{o}} 1$, julho, 2018 
com o transtorno do espectro autista. Foram realizadas pesquisas bibliográficas que indicaram o valor que o uso da tecnologia poderia trazer no âmbito educacional de crianças autistas, com foco principal na personalização da interface a fim de motivar e manter o interesse da criança, de acordo com suas preferências e características pessoais. $\mathrm{O}$ estudo dos trabalhos relacionados mostrou que essa seria uma oportunidade importante, explorando a criação de um sistema com suporte a novas tecnologias, de forma a oferecer uma interface responsiva, com suporte mobile e touchscreen, características ainda pouco exploradas em trabalhos na área.

Para pôr em prática a implementação do sistema, foi desenvolvido um protótipo de aplicação web para uso em desktop e mobile. A aplicação foi testada e validada através de um cenário de simulação, além de contar com o auxílio de cinco voluntários com experiência na área de autismo que testaram a interface em tempo real e deram seu feedback via um questionário. Os resultados mostraram que mesmo ainda não sendo uma aplicação completa, o sistema proposto pode auxiliar na resolução do problema inicialmente identificado.

A principal contribuição deste trabalho passa por ilustrar uma abordagem para a educação de crianças diagnosticadas com autismo, levando em consideração suas dificuldades, seus interesses e suas preferências, mantendo-as motivadas no processo de aprendizado e melhorando a qualidade de vida não somente destas, mas também de seus respectivos familiares. Com base neste trabalho, surgem algumas oportunidades para trabalhos futuros, podendo-se utilizar o modelo proposto e também o protótipo desenvolvido como base para o desenvolvimento de outras aplicações. Funcionalidades adicionais como delegar tarefas educativas diárias de acordo com o humor diário da criança, gerar estatísticas de uso da aplicação e trabalhar a recomendação de conteúdo fazendo a análise das estatísticas geradas são algumas possibilidades.

\section{REFERÊNCIAS}

AS, Bondy. PECS: Potential Benefits and Risks. The Behavior Analyst Today, v. 2, p. 127-132, 2001.

BERNARD-OPITZ, V.; SRIRAM, N.; NAKHODA-SAPUAN, S. Enhancing social problem solving in children with autism and normal children with computer-assisted instruction. Journal of Autism and Developmental Disorder, v. 31, p. 377-384, 2001.

DAVIS, F. D. Perceived usefulness, perceived ease of use, and user acceptance of information technology. MIS quarterly, p. 319-340, 1989.

DELLER, Robert. A curriculum guide for teaching young mentally handicapped children. Malaysian Care, 1993.

DEY, Anind K; SALBER, Daniel; ABOWD, Gregory D. A conceptual framework and a toolkit for supporting the rapid prototyping of context-aware application. Human Computer Interaction, v. 16, p. 10, 2001.

Department Of Health (UK). Basic Guidelines for People Who Commission Easy Read Inf ormation. Department of Health, UK, 2009.

FILIPEK, et al. The screening and diagnosis of autistic spectrum disorders. Journal of Autism and Developmental Disorders, v. 29, n. 6, p. 439-484, 1999.

FITZGERALD, M. Creativity psychosis autism and the social brain. InTech, 2011.

GELLES, Solange Depera. Manual da pedagogia das cores. 2 ed. All Print, 2012.

ISMAIL, A.; OMAR, N.; ZIN, A. Mohd. Developing learning software for children with learning disabilities through Block-Based development approach. International Conference on Electrical Engineering and Informatics, Selangor, p. 299-303, 2009.

L, Kanner. Autistic disturbances of affective contact. Nervous Child: Journal of Psychopathology, Psychotherapy, Mental Hygiene, and Guidance of the Child, v. 2, p. 217-250, 1943.

V. $16 \mathrm{~N}^{\mathrm{o}} 1$, julho, 2018 
LEO, Gianluca De; LEROY, Gondy. Smartphones to facilitate communication and improve social skills of children with severe autism spectrum disorder: special education teachers as proxies. In Proceedings of the 7th international conference on Interaction design and children (IDC '08), ACM, New York, NY, USA, p. 45-48, 2008.

LIKERT, R. A Technique for the Measurement of Attitudes. Archives of Psychology, v. 22, n. 140, p. 1-55, 1932.

MINTZ, J. Additional Key Factors Mediating the Use of a Mobile Technology Tool Designed to Develop Social and Life Skills in Children with Autism Spectrum Disorders: Evaluation of the 2nd HANDS Prototype. Computers \& Education, v. 63, p. 17-27, 2013.

MORRIS, Robert R.; KIRSCHBAUM, Connor R.; PICARD, Rosalind W. Broadening accessibility through special interests: a new approach for software customization. In Proceedings of the 12th international ACM SIGACCESS conference on Computers and accessibility (ASSETS '10). ACM, New York, NY, USA, p. 171 $178,2010$.

PAVLOL, N. User Interface for People with Autism Spectrum Disorders. Journal of Software Engineering and Applications, v. 7, n. 2, p. 128-134, 2014.

POWELL, S. The use of computers in teaching people with autism. autism on the agenda. In Papers from a National Autistic Society Conference, p. 128-132, 1996.

PUTNAM, Cynthia; CHONG, Lorna. Software and technologies designed for people with autism: what do users want? In Proceedings of the 10th international ACM SIGACCESS conference on Computers and accessibility (Assets '08). ACM, New York, NY, USA, p. 3-10, 2008.

R.R, Jordan; Powell S. Understanding and Teaching Children with Autism. Chichester: Wiley, 1995.

RAHMAN, M. R. et al. A-class: A classroom software with the support for diversity in aptitudes of autistic children. IEEE Symposium on Computers \& Informatics, Kuala Lumpur, p. 727-731, 2011.

SATYANARAYANAN, M. Pervasive computing: vision and challenges. IEEE Personal Communications, v. 8, n. 4, p. 10-17, 2001.

SILVA, M. L. D. et al. A Web-based Application to Address Individual Interests of Children with Autism Spectrum Disorders. Procedia Computer Science, v. 14, p. 20-27, 2012.

SILVA, Margarida Lucas Da; GONÇALVES, Daniel; SILVA, Hugo. User-tuned Content Customization for Children with Autism Spectrum Disorders. Procedia Computer Science, v. 27, p. 441-448, 2014.

UNGER, R.; CHANDLER, C. O guia para projetar UX: a experiência do usuário (UX) para projetistas de conteúdo digital, aplicações e web sites. Rio de Janeiro: Alta Books, 2009.

WHALEN, C. et al. Behavioral improvements associated with computer-assisted instruction for children with developmental disabilities. The Journal of Speech and Language Pathology - Applied Behavior Analysis, v. 1, n. 1, p. 11-26, 2006.

WILLIAMS, C. et al. Do children with autism learn to read more readily by computer assisted instruction or traditional book methods? A pilot study. Autism, v. 6, n. 1, p. 71-91, mar. 2002.

YOON, C; KIM, S. Convenience and TAM in a ubiquitous computing environment: The case of wireless LAN. Electronic Commerce Research and Applications, v. 6, n. 1, p. 102-112, mar. 2007. 